OPEN ACCESS

Edited by:

Marcela Alejandra Michaut,

CONICET Dr. Mario H. Burgos Institute of Histology and Embryology

(IHEM), Argentina

Reviewed by:

Mircea Dumitru Croitoru,

George Emil Palade University of Medicine, Pharmacy, Science, and Technology of Târgu Mureş,

Romania

Kenji Miyado,

National Center for Child Health and Development (NCCHD), Japan

${ }^{*}$ Correspondence:

Cristina Soriano-Úbeda cmsu1@um.es Carmen Matás cmatas@um.es

Specialty section:

This article was submitted to Molecular and Cellular Reproduction, a section of the journal Frontiers in Cell and Developmental Biology

Received: 28 December 2020 Accepted: 08 March 2021 Published: 14 April 2021

Citation:

Staicu F-D, Canha-Gouveia A Soriano-Úbeda C, Martínez-Soto JC, Adoamnei E, Chavarro JE and

Matás C (2021) Nitrite and Nitrate Levels in Follicular Fluid From Human Oocyte Donors Are Related to Ovarian

Response and Embryo Quality. Front. Cell Dev. Biol. 9:647002. doi: 10.3389/fcell.2021.647002

\section{Nitrite and Nitrate Levels in Follicular Fluid From Human Oocyte Donors Are Related to Ovarian Response and Embryo Quality}

\author{
Florentin-Daniel Staicu ${ }^{1,2}$, Analuce Canha-Gouveia ${ }^{1,2}$, Cristina Soriano-Úbeda ${ }^{1,2,3 *}$, \\ Juan Carlos Martínez-Soto ${ }^{2,4}$, Evdochia Adoamnei ${ }^{2,5}$, Jorge E. Chavarro ${ }^{6,7,8}$ and \\ Carmen Matás ${ }^{1,2 *}$ \\ 1 Department of Physiology, Faculty of Veterinary Science, International Excellence Campus for Higher Education \\ and Research (Campus Mare Nostrum), University of Murcia, Murcia, Spain, ${ }^{2}$ Biomedical Research Institute of Murcia \\ (IMIB), Murcia, Spain, ${ }^{3}$ Department of Veterinary and Animal Sciences, University of Massachusetts Amherst, Amherst, MA, \\ United States, ${ }^{4} \mathrm{IVI}$-RMA Global, Murcia, Spain, ${ }^{5}$ Department of Nursing, School of Nursing, University of Murcia, Murcia, \\ Spain, ${ }^{6}$ Department of Nutrition, Harvard T.H. Chan School of Public Health, Boston, MA, United States, ${ }^{7}$ Department \\ of Epidemiology, Harvard T.H. Chan School of Public Health, Boston, MA, United States, ${ }^{8}$ Channing Division of Network \\ Medicine, Brigham and Women's Hospital, Harvard Medical School, Boston, MA, United States
}

Nitric oxide, a key regulatory molecule in the follicular fluid, has been suggested as a possible biomarker to predict ovarian response in stimulated cycles and the potential of the retrieved oocytes for developing high-quality embryos. Nevertheless, a consensus on whether or not nitric oxide can help in this context has not been reached. We simultaneously measured the oxidation products of nitric oxide, nitrite, and nitrate, via high-performance liquid chromatography (HPLC)-UV in follicular fluid samples from 72 oocyte donors. We found no associations of follicular fluid nitrite, nitrate, total nitric oxide, or nitrate/nitrite ratio with total or metaphase II (MII) oocyte yield. However, nitrite and nitrate levels were related to the yield of MII oocytes when this outcome was expressed as a proportion of all oocytes retrieved. The adjusted MII proportion in the lowest and highest nitrite levels were 68\% (58-77\%) and 79\% (70-85\%), respectively ( $p$, linear trend $=0.02$ ), whereas the adjusted MII proportion in extreme tertiles of nitrate levels were 79\% (70-85\%) and 68\% (57-77\%) ( $p$, linear trend = 0.03). In addition, nitrate levels showed a suggestive inverse correlation with embryos with maximum or high potential of implantation $(p=0.07$ ). These results suggest that the follicular fluid concentrations of nitrite and nitrate may be a useful tool in predicting how healthy oocyte donors respond to superovulation and the implantation potential of the embryos produced from their oocytes.

Keywords: nitric oxide, nitrite, nitrate, follicular fluid, oocyte, embryo

\section{INTRODUCTION}

A large number of couples of reproductive age struggle with infertility issues, the causes of which are not always clear (Agarwal and Allamaneni, 2004). For this reason, several studies have tried to identify new biochemical markers that can affect gamete and embryo quality and may predict the outcome of infertility treatment with in vitro fertilization (IVF) (Yalçınkaya et al., 2013). 
Nitric oxide (NO) has emerged as a candidate predictor of ovarian response and IVF outcomes (Barroso et al., 1999). Apart from being a well-known regulator of vasodilation and neurotransmission (Pacher et al., 2007), NO has also been linked to the granulosa cell function (Basini et al., 1998), meiotic resumption (Romero-Aguirregomezcorta et al., 2014), and prevention of oocyte aging (Goud et al., 2005), as well as ovulation (Anteby et al., 1996). However, when investigating the relation of intrafollicular levels of NO with oocyte recruiting, fertilization potential, embryo quality, implantation, and pregnancy rates in patients undergoing IVF, the results are contradictory. On one hand, evidence suggests that NO levels in the follicular fluid (FF) are inversely associated with the fertilization of mature oocytes and the ability of the subsequent embryo to cleave normally (Barrionuevo et al., 2000). Furthermore, a negative correlation between FF NO levels and embryo morphology was identified (Barroso et al., 1999; Battaglia et al., 2006). On the other hand, other studies found no differences in relation to oocyte and embryo quality (Lee et al., 2000; Yalçınkaya et al., 2013). The relationship between FF, NO, and pregnancy outcome is also uncertain (Lee et al., 2000; Kim et al., 2004; Yalçınkaya et al., 2013).

The controversy among these data might reside in the unstable nature of NO, which makes its direct measurement difficult (Manau et al., 2000). NO has a short biological half-life that can be influenced by different factors, such as its concentration, the presence or not of NO scavengers (Hakim et al., 1996), and the cellular redox state (Rosselli et al., 1998). NO itself can act as a free radical scavenger and prevent cell toxicity by inactivating the superoxide anion. Under specific conditions, however, this interaction can generate peroxynitrite, a potent oxidant (Rosselli et al., 1998). Additionally, NO is oxidized in blood and tissues leading to the formation of two stable end-products, nitrite $\left(\mathrm{NO}_{2}\right)$ and nitrate $\left(\mathrm{NO}_{3}\right)$ (Lundberg et al., 2008), which are a suitable to quantify indirectly NO synthesis (Romitelli et al., 2007). However, the methods for $\mathrm{NO}_{2}$ and $\mathrm{NO}_{3}$ detection have limitations such as the time required for the analysis, interference from other ions, or the difficulty to detect $\mathrm{NO}_{2}$ and $\mathrm{NO}_{3}$ at the same time and in minor concentrations (Croitoru, 2012). The simultaneous detection of low $\mathrm{NO}_{2}$ and $\mathrm{NO}_{3}$ concentrations has been described in mammal blood, urine, and in plant samples by high-performance liquid chromatography (HPLC)UV (Croitoru, 2012). This study aims, first of all, to apply this method with some modifications to determine the FF levels of $\mathrm{NO}_{2}, \mathrm{NO}_{3}$, total $\mathrm{NO}(\mathrm{NOx})$, and $\mathrm{NO}_{3} / \mathrm{NO}_{2}$ ratio in oocyte donors. Subsequently, it aims to further clarify any associations between these parameters, ovarian response, and quality of the embryos derived from oocytes of healthy women undergoing ovarian stimulation as part of their participation in an oocyte donation program.

\section{MATERIALS AND METHODS}

\section{Ethics}

This study was approved by the Ethics Review Committee of CEIC Hospital General Universitario Jose Maria Morales
Meseguer (Murcia, Spain) (Approval No. EST: 06/17) and registered at https://clinicaltrials.gov/ (ID: NCT03307655). Women participating in the gamete donation program at IVI-RMA Global (Murcia, Spain) were invited to participate in the study. All the donors who accepted provided their written informed consent.

\section{Clinical and Lifestyle-Related Data}

The following data regarding the donation cycle were collected from IVI-RMA Global (Murcia, Spain) for all participants: total and metaphase II (MII) oocyte yield, number of stimulation days, 17ß-estradiol (E2) peak, total dose of follicle-stimulating hormone (FSH), oocyte fate (fresh transfer, vitrified and mixed), and quality of the blastocyst derived from their oocytes. Donors who enrolled in the study were asked to report their demographic and lifestyle characteristics over the previous year such as age, body mass index (BMI), sleep time, diet, coffee and alcohol consumption, smoking history, leisure physical activity, and sedentary behavior. The smoking history was classified in three different categories (ever smoker, self-reported exposure to secondhand smoke) and physical activity in Leisure time vigorous/moderate physical activities, hours/week, and Sedentary behavior hours/week. BMI was calculated as weight $(\mathrm{kg})$ divided by height squared $\left(\mathrm{m}^{2}\right)$. The sleep was evaluated distinguishing the hours of sleep per day and the napping time. The mean number of servings per week of several food items was recorded using a food frequency questionnaire adapted to meet specific study objectives. Special attention was given to specific vegetables known by its high mean nitrate content, like spinach (Lidder and Webb, 2013). Later, according to Panagiotakos et al. (2007), all the listed food items were rearranged in different groups presumed to be close to Mediterranean dietary pattern, namely, the non-refined cereals, fruits, vegetables, legumes, olive oil, fish, and potatoes group. For example, the vegetables group was formed by the sum of cabbage, cauliflower, broccoli, cooked or raw, artichokes, asparagus, carrot, spinach or cooked chard, lettuce, endives, onion, cooked green beans, aubergines, zucchini, cucumbers, peppers, boiled corn, and tomato. A different score scale ( $0-5$ for never, rare, frequent, very frequent, weekly, and daily consumption) was assigned to each group. Subsequently, the Mediterranean diet score (on a scale of 0-55) was calculated by summing up the corresponding scores of these groups. To calculate the total intake, the alcohol content for specific items was summed and multiplied by weights proportional to the frequency of use of each item.

\section{Superovulation of Oocyte Donors and Oocyte Retrieval}

Superovulation was achieved by means of a human recombinant follicle-stimulating hormone and a gonadotrophin-releasing hormone antagonist, as previously described (Melo et al., 2009). When the follicles reached an average diameter of 17.5$18 \mathrm{~mm}$, a gonadotropin-releasing hormone $(\mathrm{GnRH})$ agonist was administered to induce ovulation (Melo et al., 2009). Approximately $36 \mathrm{~h}$ later, dominant follicles were punctured transvaginally under ultrasound guidance, and FF was aspirated 
together with the oocyte in Sequential Fert $^{\text {TM }}$ medium $\left(\right.$ Origio $^{\circledR}$, CooperSurgical Fertility and Genomic Solutions, Målov, Denmark).

\section{Intracytoplasmic Sperm Injection and Embryo Culture}

After retrieval, the oocytes of 72 donors were washed in Sequential Fert ${ }^{\mathrm{TM}}$ medium. The removal of cumulus cells was performed by gently pipetting the oocytes in a solution of 80 $\mathrm{IU} / \mathrm{ml}$ hyaluronidase in Sequential Fert ${ }^{\mathrm{TM}}$ medium. Oocytes were cultured in fertilization medium $\left(\mathrm{Gems}^{\circledR}{ }^{\circledR}\right.$, Genea Biomedx, Sydney, NSW, Australia) at $5 \% \mathrm{CO}_{2}, 37^{\circ} \mathrm{C}$, and atmospheric $\mathrm{O}_{2}$ for $3 \mathrm{~h}$. After that, the oocytes were placed in a microdrop of fertilization medium for performing intracytoplasmic sperm injection (ICSI). Sperm samples were obtained from ejaculates without oligo-asteno-teratozoospermia and selected by density gradient 45/90\% (SIP100, Sil-Select Plus, FERTIPRO NV, Belgium), diluted in a solution of $10 \%$ polyvinylpyrrolidone (PVP) in Sequential Fert ${ }^{\mathrm{TM}}$ medium, and placed in a microdrop for performing ICSI. Immediately after ICSI, the zygotes were transferred to the pre-equilibrated embryo culture medium Cleavage Medium ${ }^{\mathrm{TM}}{ }^{\left(G e m s^{\circledR}\right.}{ }^{\circledR}$, Genea Biomedx, Sydney, NSW, Australia) and covered with mineral oil (LifeGuard ${ }^{\circledR}$, Genomicks Sdn Bhd, Petaling Jaya, Malaysia) and cultured for 5 days. Several parameters were analyzed in the embryos: the fertilization rate (\%), embryo rate (\%) - percentage of embryos that cleaved - and quality of the embryos at day 5. Embryo quality was evaluated by morphological scoring at day 5 of culture (blastocyst stage) by experienced technicians according to the standardized criteria of the Spanish Association for the Study of the Biology of Reproduction (ASEBIR) (Hurtado de Mendoza et al., 2015; Cuevas Saiz et al., 2018; Figure 1). The morphological parameters considered for this evaluation were the size and cellular cohesion of the blastomeres in the inner cell mass (ICM) and the homogeneity, cohesion, and number of cells of the trophectoderm (TE). According to these criteria, embryos were classified in four grades (A-D) summarized in Table $\mathbf{1}$. Degenerated or dead oocytes and embryos were excluded from the study. The retrieved oocytes of 72 donors were used for one respective recipient. However, since three of those 72 donors had donated an elevated amount of MII oocytes, their oocytes were used for more than one recipient and, consequently, were inseminated with spermatozoa of different men. Therefore, we present a total $n=75$ of embryo data.

\section{FF Samples}

Following the oocyte retrieval, the FF samples were centrifuged at $4^{\circ} \mathrm{C}$ during $15 \mathrm{~min}$ at $1500 \times g$. The supernatant was filtered using 0.22- $\mu \mathrm{m}$ filter units (Merck KGaA, Darmstadt, Germany) to remove cellular debris, then aliquoted and stored at $-20^{\circ} \mathrm{C}$ until use. Before and after centrifugation, an aliquot from 26 FF samples was used to determine hemoglobin ( $\mathrm{Hb}$ ) levels with a HemoCue Plasma/Low Hb System (Ängelholm, Sweden). This was done to ensure that the puncture procedure did not affect the $\mathrm{Hb}$ levels in the fluid, which, if present after the
FF has been processed, can cause $\mathrm{NO}_{2}$ reduction or oxidation (Lundberg et al., 2008).

\section{$\mathrm{NO}_{2}$ and $\mathrm{NO}_{3}$ Measurements in FF}

Nitrite and $\mathrm{NO}_{3}$ levels in FF samples were measured by HPLCUV, using a method previously described by Croitoru (2012) with some changes, namely, in the column used, flowrate, duration, and without any derivatization step. In detail, the analysis was carried out on an Agilent 1100 Series HPLC System (Agilent Technologies, Santa Clara, CA, United States) equipped with a thermostated microwell plate autosampler, a quaternary pump, and a multiple wavelength absorbance detector. Standards and samples $(40 \mu \mathrm{l})$ were injected into an Agilent Zorbax Eclipse XDB-C18 HPLC column $(4.6 \mathrm{~mm} \times 150 \mathrm{~mm}, 5 \mu \mathrm{m})$, thermostated at $25^{\circ} \mathrm{C}$, and eluted at a flowrate of $400 \mu \mathrm{l} / \mathrm{min}$ during the whole separation. Mobile phase A consisted of $5 \mathrm{mM}$ tetrabutylammonium hydroxide $\mathrm{pH} 2.5$ (Sigma-Aldrich Química S.A., Madrid, Spain) and 8\% v/v acetonitrile (VWR Chemicals, Barcelona, Spain) in water, while mobile phase B was methanol (VWR Chemicals, Barcelona, Spain). The gradient elution program was $100 \%$ solvent A for $10 \mathrm{~min}$, a linear gradient from 0 to $50 \%$ solvent $B$ for $20 \mathrm{~min}$, and $10 \mathrm{~min}$ at constant $100 \%$ solvent B. The column was equilibrated with the starting composition of the mobile phase for $15 \mathrm{~min}$ before each analytical run. The $206 \mathrm{~nm}$ absorbance signal was recorded.

High-performance liquid chromatography standards were prepared in Milli-Q water using reagent grade sodium nitrite and sodium nitrate (Sigma-Aldrich Química S.A., Madrid, Spain). Both standards were prepared at a concentration of $1 \mathrm{mM}$, and serial dilutions from 100 to $0.1 \mu \mathrm{M}$ were used to obtain the calibration curve for the analysis. FF samples were thawed and filtered through Amicon $3 \mathrm{~K}$ centrifugal units (Merck KGaA, Darmstadt, Germany) to eliminate proteins. The centrifugal units were first rinsed with Milli-Q water to equilibrate the membrane and then centrifuged for $10 \mathrm{~min}$ at $14,000 \times \mathrm{g}$. The receiver tube was replaced with a new one, and $400 \mu \mathrm{l}$ of the sample was added to the centrifugal unit and centrifuged for $60 \mathrm{~min}$ at $14,000 \times \mathrm{g}$. The clean filtrates were used for the analysis. The UV chromatograms at $206 \mathrm{~nm}$ from both standards and samples were analyzed with Chemstation Rev B.01.03.SR2 (Agilent Technologies, Santa Clara, CA, United States). The $\mathrm{NO}_{2}$ and $\mathrm{NO}_{3}$ peak areas in the standard solutions were used for the calculation of the calibration curve, from which the concentration in samples was obtained. The measurements were performed in duplicate, and the sum of mean values of $\mathrm{NO}_{2}$ and $\mathrm{NO}_{3}$ levels was used to calculate NOx concentration (RatajczakWrona et al., 2013). The ratio between mean values of $\mathrm{NO}_{3}$ and $\mathrm{NO}_{2}$ levels was also determined.

\section{Statistical Analysis}

Descriptive statistics were calculated for demographic, lifestyle, first donation cycle characteristics in the entire cohort, plus embryo production and quality by percentiles of $\mathrm{NO}_{2}, \mathrm{NO}_{3}$, $\mathrm{NOx}$, and $\mathrm{NO}_{3} / \mathrm{NO}_{2}$ ratio. The presence of any associations was evaluated by using ANOVA and chi-square tests for continuous and categorical variables, respectively. These data were presented as mean (standard deviation, SD) or number of women (\%). 

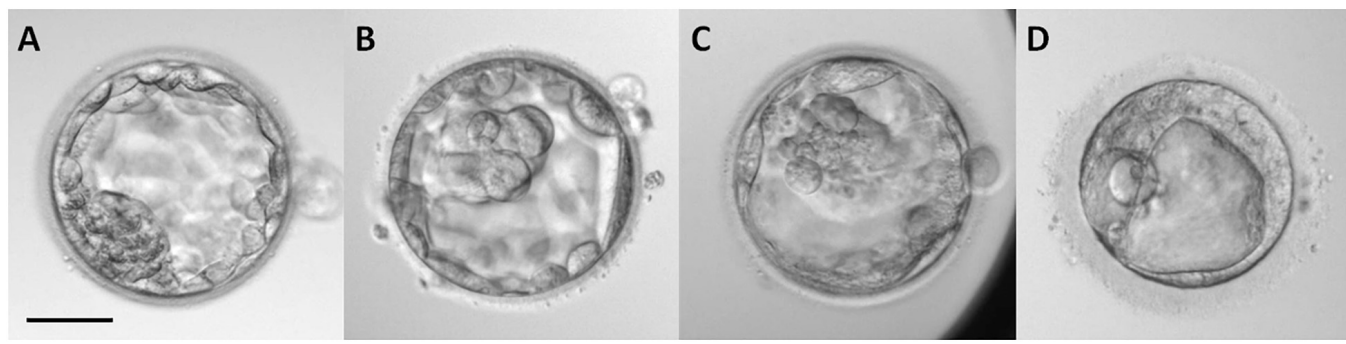

FIGURE 1 | Morphological classification of blastocysts in day 5 of in vitro development according to the criteria established by the Spanish Association for the Study of the Biology of Reproduction (ASEBIR) based on the morphological evaluation of the inner cell mass (ICM) and trophectoderm (TE) (Hurtado de Mendoza et al., 2015; Cuevas Saiz et al., 2018). The implantation potential of blastocysts according to this classification is established as follows: grade (A), maximum; grade (B), high; grade (C), medium; grade (D), low (see Table 1). Scale bar represents $25 \mu \mathrm{m}$.

TABLE 1 | Spanish Association for the Study of the Biology of Reproduction (ASEBIR) scoring for blastocyst stage in day 5 of embryo development according to morphological evaluation of the inner cell mass (ICM) and trophectoderm (TE) (Hurtado de Mendoza et al., 2015; Cuevas Saiz et al., 2018).

\begin{tabular}{lccccc}
\hline & \multicolumn{2}{c}{ Inner cell mass (ICM) } & Trophectoderm (TE) & Embryo quality & Potential of implantation \\
\cline { 2 - 4 } Embryo grade & Blastomeres size & Cellular cohesion & & & \\
\hline A & $3800-1900 \mu \mathrm{m}^{2}$ & Compacted & Homogeneous, cohesive, many cells & 1st or optimus \\
B & $3800-1900 \mu \mathrm{m}^{2}$ & Loose & Homogeneous, not many cells & 2nd or good & Maximum \\
C & $1900 \mu \mathrm{m}^{2}$ & Any & High & Med medium \\
D & Ongoing degeneration & Any & Ongoing degeneration & 4th or bad & Low \\
E (excluded) & Degenerated & Any & Degenerated & Sth or degenerated
\end{tabular}

Multivariable mixed Poisson and logistic regression models with random slopes to account for repeated observations within a woman were used to compare total and MII oocyte yields, as well as the proportion of MII oocytes, across tertiles of $\mathrm{NO}_{2}$, $\mathrm{NO}_{3}, \mathrm{NOx}$, and $\mathrm{NO}_{3} / \mathrm{NO}_{2}$ ratio. Categorical covariables were included using indicators for missing covariates when necessary. Multivariable-adjusted models included terms for age, body mass index, sleep time, coffee intake, smoking history, and leisure physical activity as potential confounders of the relation between NO metabolites and measures of ovarian response to hyperstimulation. Correlations (Spearman's rho) between NOx levels and dietary pattern variables were also evaluated, namely, the Mediterranean diet score, the consumption of vegetables in general or specifically the ones with high NOx content like spinach. Tests for linear trend were conducted by modeling the tertiles of each metabolite, using the median analyte concentration values in each tertile, as a continuous linear term. For embryo development and quality, continuous variables were summarized by arithmetic mean, SD, range, and selected percentiles, including the median. Spearman's rank correlation coefficients were used to explore the relationship between $\mathrm{NO}_{2}$, $\mathrm{NO}_{3}, \mathrm{NOx}, \mathrm{NO}_{3} / \mathrm{NO}_{2}$ ratio, and embryonic parameters. All analyses were performed in SAS 9.4 (SAS Institute) and IBM SPSS 25.0 (IBM Corporation, Armonk, NY, United States).

\section{RESULTS}

Seventy-two women participating in the oocyte donation program at IVI-RMA Global Murcia (Spain), between
February 2017 and September 2018, were included in our cohort. FF was obtained at oocyte retrieval in 93 oocyte donation cycles to measure $\mathrm{NO}_{2}$ and $\mathrm{NO}_{3}$ levels by HPLCUV (Figure 2). The first peak, located at $10.2 \mathrm{~min}$ was identified as $\mathrm{NO}_{2}$, while the peak at $31.6 \mathrm{~min}$ was identified as $\mathrm{NO}_{3}$. When analyzing the chromatogram from different $\mathrm{FF}$ samples, we observed the same peaks with the same retention times (Figure 2).

The FF concentrations of $\mathrm{NO}_{2}, \mathrm{NO}_{3}, \mathrm{NOx}$, and the $\mathrm{NO}_{3} / \mathrm{NO}_{2}$ ratio are reported in Table 2. $\mathrm{NO}_{2}$ levels ranged from 0.7 to $96.1 \mu \mathrm{M}, \mathrm{NO}_{3}$ levels ranged from 4.9 to $39.7 \mu \mathrm{M}$, NOx levels ranged from 5.6 to $109.5 \mu \mathrm{M}$, and $\mathrm{NO}_{3} / \mathrm{NO}_{2}$ ratio ranged from 0.1 to 31.5. $\mathrm{NO}_{2}$ and $\mathrm{NO}_{3}$ concentrations were unrelated to each other $(r=-0.01) . \mathrm{NO}_{2}$ was positively correlated with $\mathrm{NOx}$ and negatively correlated with the $\mathrm{NO}_{3} / \mathrm{NO}_{2}$ ratio (Table 2).

No significant differences were found when analyzing the following variables across tertiles of $\mathrm{NO}_{2}$ and $\mathrm{NO}_{3}$ : age at egg donation, BMI, Mediterranean diet score, coffee and occasional alcohol intake, secondhand exposure to smoke, and sedentary behavior (Table 3). On the other hand, women with higher FF $\mathrm{NO}_{2}$ levels were more likely to sleep less [mean (SD) of 7.0 (2.0) $\mathrm{h} /$ day] and spend more time per week in leisure activities [mean (SD) of $3.7(6.0) \mathrm{h} /$ week]. Moreover, higher $\mathrm{NO}_{2}$ and $\mathrm{NO}_{3}$ concentrations were present in donors who smoked (22.2 and $23.6 \%$, respectively), either at the time of the study or in the past. No significant correlations between NOx levels and vegetables intake were observed (Supplementary Tables 1, 2). The characteristics of the first donation cycle, such as the number of stimulation days, total dose of FSH, and the oocyte fate, were similar across tertiles of $\mathrm{NO}_{2}$ and $\mathrm{NO}_{3}$, but donors with low $\mathrm{FF}$ 


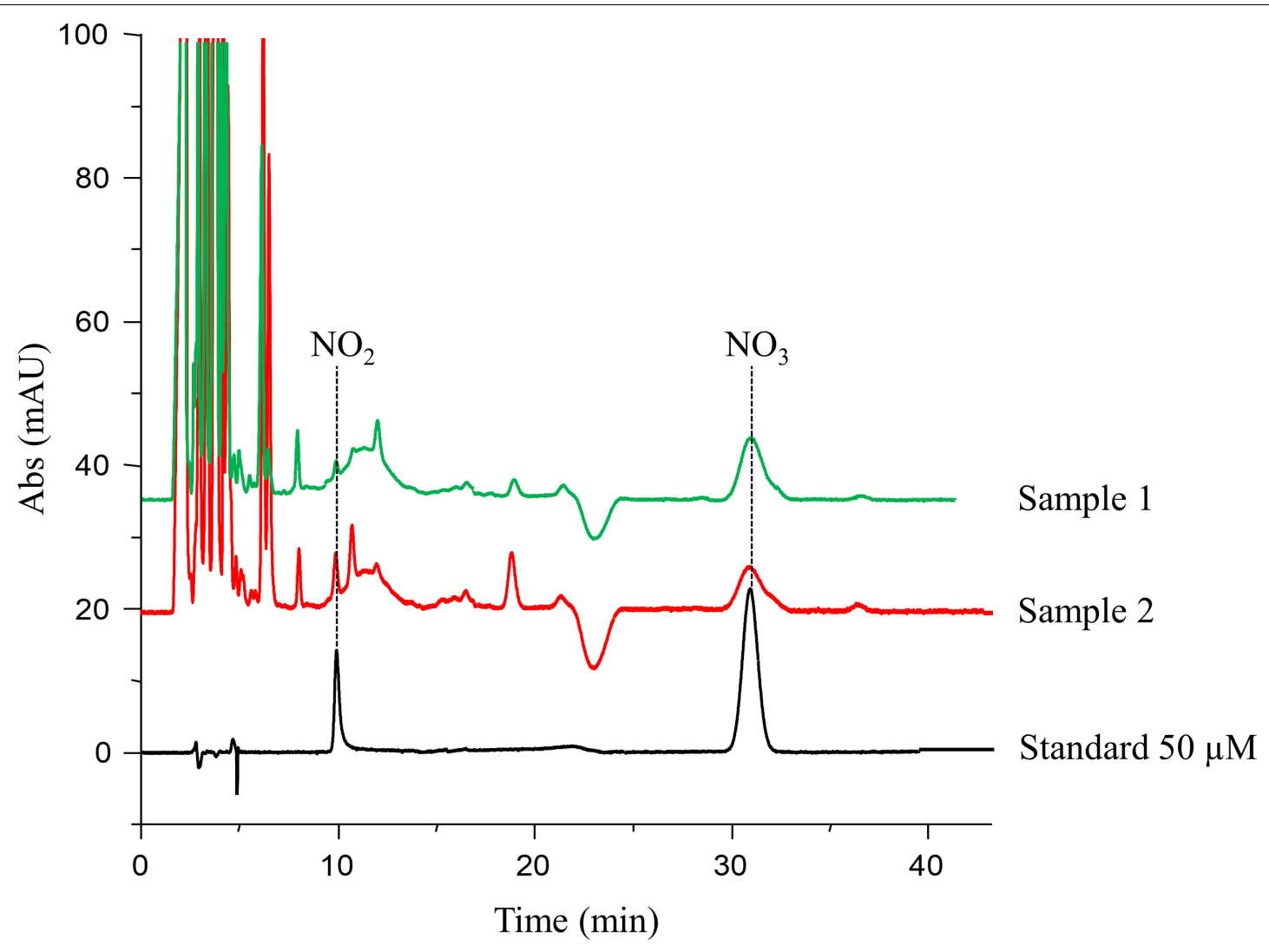

FIGURE 2 | High-performance liquid chromatography (HPLC)-UV chromatogram. The peak located at 10.2 min was identified as nitrite ( $\mathrm{NO}_{2}$ ), while the peak at $31.6 \mathrm{~min}$ was identified as nitrate $\left(\mathrm{NO}_{3}\right)$. The peak areas of each compound in the standard solutions were used for the calculation of the calibration curve, from which the concentrations in follicular fluid samples (e.g., samples 1 and 2) were obtained.

TABLE 2 | Follicular fluid levels of nitrite $\left(\mathrm{NO}_{2}\right)$, nitrate $\left(\mathrm{NO}_{3}\right)$, total nitric oxide $(\mathrm{NOx}), \mathrm{NO}_{3} / \mathrm{NO}_{2}$ ratio, and Pearson correlation coefficients between these parameters.

\begin{tabular}{|c|c|c|c|c|c|c|c|}
\hline & \multirow[b]{2}{*}{ Minimum } & \multirow[b]{2}{*}{ Maximum } & \multirow[b]{2}{*}{ Mean (SD) } & \multicolumn{4}{|c|}{$\boldsymbol{R}$} \\
\hline & & & & $\mathrm{NO}_{2}$ & $\mathrm{NO}_{3}$ & NOx & $\mathrm{NO}_{3} / \mathrm{NO}_{2}$ ratio \\
\hline $\mathrm{NO}_{2}$ & 0.7 & 96.1 & 14.7 (12.3) & 1.00 & -0.01 & 0.89 & -0.40 \\
\hline $\mathrm{NO}_{3}$ & 4.9 & 39.7 & $17.3(6.3)$ & & 1.00 & 0.45 & 0.14 \\
\hline $\mathrm{NOx}$ & 5.6 & 109.5 & $31.9(13.7)$ & & & 1.00 & -0.29 \\
\hline $\mathrm{NO}_{3} / \mathrm{NO}_{2}$ ratio & 0.1 & 31.5 & $2.6(4.4)$ & & & & 1.00 \\
\hline
\end{tabular}

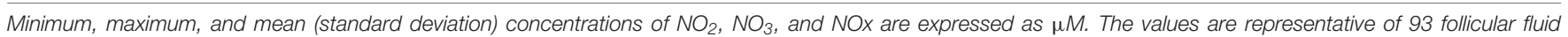
samples.

$\mathrm{NO}_{2}$ levels likely had a higher E2 peak [mean (SD) of 2004.2 (1140.8) pg/mL].

Follicular fluid $\mathrm{NO}_{2}$ and $\mathrm{NO}_{3}$ were unrelated to total or mature oocyte yield (Table 4). The multivariable-adjusted MII yield (95\% CI) for women in the lowest and highest tertiles of $\mathrm{NO}_{2}$ was $12.4(10.2,15.1)$ and $13.2(10.9,16.0)$ (p, linear trend $=0.38)$ and $14.1(11.7,17.1)$ and $12.2(9.9,15.0)$ for $\mathrm{NO}_{3}$ (p, linear trend $=0.14$ ), respectively. When MII oocytes were considered as the proportion of total oocytes, however, the proportion of MII oocytes increased with increasing $\mathrm{FF} \mathrm{NO}_{2}$ levels but decreased with increasing $\mathrm{NO}_{3}$ levels. The adjusted proportion (95\% CI) of MII oocytes for women in the lowest and highest FF levels of $\mathrm{NO}_{2}$ were $68 \%(58-77 \%)$ and $79 \%$ $(70-85 \%)(\mathrm{p}$, linear trend $=0.02)$, whereas the proportion of MII oocytes for women in extreme tertiles of FF $\mathrm{NO}_{3}$ levels were $79 \%(70-85 \%)$ and $68 \%(57-77 \%)(p$, linear trend $=0.03)$. $\mathrm{NOx}$ and the $\mathrm{NO}_{3} / \mathrm{NO}_{2}$ ratio were unrelated to the total and mature oocyte yield, whether expressed in absolute or relative terms (Table 4). Summary statistics for embryo development and quality are shown in Table 5.

A suggestive (borderline) inverse correlation between $\mathrm{NO}_{3}$ levels and the number of embryos type $\mathrm{A}+\mathrm{B}$ on day $5(p=0.07)$ and embryo cleavage rate on day $5(p=0.08)$ were observed (Figure 3 and Table 6). 


\section{DISCUSSION}

One of the factors associated with successful pregnancy during IVF is oocyte quality (Lee et al., 2000). The oocyte development takes place in a dynamic microenvironment, where the complex composition of the FF is very important (Vignini et al., 2008). Among other molecules, FF contains free radicals, like NO, which is actively synthesized by the granulosa cells (Lee et al., 2000). This means that NO and/or its by-products may be potential biomarkers for IVF outcome, and several studies tested this hypothesis in patients undergoing fertility treatment (Barroso et al., 1999; Barrionuevo et al., 2000; Lee et al., 2000; Battaglia et al., 2006; Vignini et al., 2008; Zhao et al., 2010; Goud et al., 2014).

The assessment of $\mathrm{NO}$ concentration can be performed indirectly by assaying two anions, i.e., $\mathrm{NO}_{2}$ and $\mathrm{NO}_{3}$ (Revelli et al., 2009). In the present work, we described how a previously validated technique for measuring simultaneously these ions (Croitoru, 2012) can also be applied in FF samples.

The levels of $\mathrm{NO}_{2}$ and $\mathrm{NO}_{3}$, described in our cohort, did not predict the total number of oocytes recovered from the donors or the MII oocytes count. Other studies described similar findings. Yalçınkaya et al. (2013) reported the absence of a correlation when evaluating the relationship between NO and IVF parameters such as the number of mature oocytes, fertilization rate, and embryo grading. Lee et al. (2000) found no significant differences between FF NO levels and the maturity and quality of the oocyte. Moreover, the $\mathrm{NO}_{2} / \mathrm{NO}_{3}$ concentrations measured in both serum and FF were not good markers of ovarian response or pregnancy in IVF cycles (Manau et al., 2000).

Interestingly, our data suggest that the FF concentrations of $\mathrm{NO}_{2}$ and $\mathrm{NO}_{3}$ are associated with the proportion of MII oocytes; particularly, the latter was related directly to $\mathrm{NO}_{2}$ levels and inversely to $\mathrm{NO}_{3}$ levels. Even though these stable ions derive from NO, previous research reports evidenced that they can be physiologically recycled to form again NO (Lundberg et al., 2008), likely to be employed for protein S-nitrosylation during maturation process of the oocyte (Romero-Aguirregomezcorta et al., 2014). According to our results, $\mathrm{FF} \mathrm{NO}_{2}$ levels appear to be more representative for $\mathrm{NO}$ formation, as shown by the Pearson correlation coefficients. The formation of $\mathrm{NO}_{2}$ takes place, for instance, by $\mathrm{NO}$ auto-oxidation, $\mathrm{NO}_{3}$ reduction (Lundberg et al., 2008), or through a reaction catalyzed by the multicopper oxidase ceruloplasmin (Shiva et al., 2006), which is also a FF component (Suchanek et al., 1990; Gonzalès et al., 1992; Gentry et al., 2000). It has been shown that its levels depend on the ovarian

TABLE 3 | Demographic, lifestyle, and first cycle characteristics by nitrite $\left(\mathrm{NO}_{2}\right)$ and nitrate $\left(\mathrm{NO}_{3}\right)$ tertiles of healthy women undergoing ovarian stimulation as part of their participation in an oocyte donation program $(n=72)$.

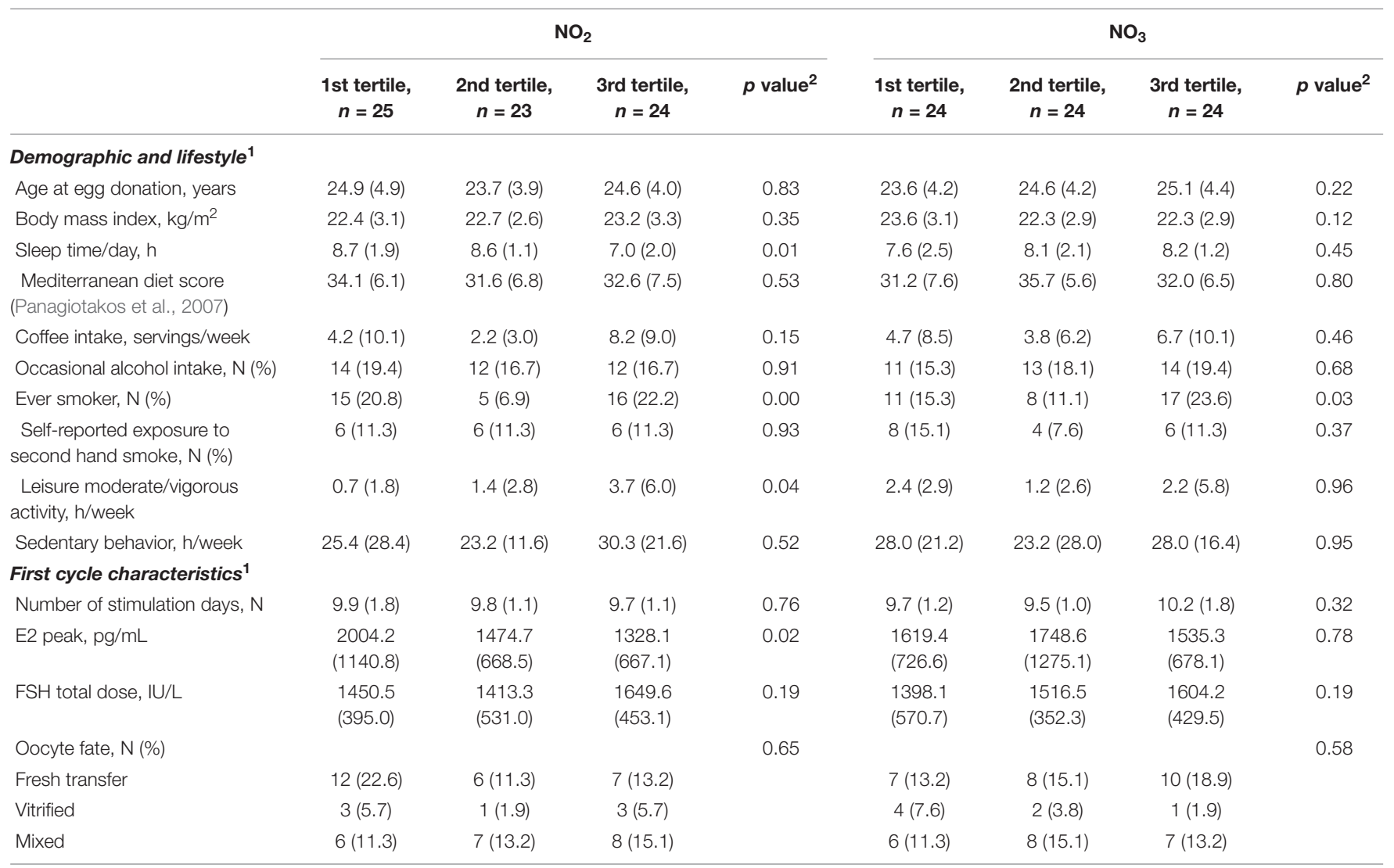

${ }^{1}$ Data are presented as mean (standard deviation) or number of women (\%).

${ }^{2}$ Differences across categories were tested using an ANOVA test for continuous variables and a chi-square test for categorical variables. 
stimulation protocol (Suchanek et al., 1990), and it was described as an indicator of oocyte maturation, since the ceruloplasmin concentration was higher in follicles containing a mature egg (Gulamali-Majid et al., 1987) and that later underwent cleavage (Gonzalès et al., 1992). $\mathrm{NO}_{2}$ formation via ceruloplasmin also produces nitrous acid (Shiva et al., 2006). Both these species can be converted back into NO in the presence of ascorbate (reviewed by Lundberg et al., 2008), which has been identified in the FF (Cigliano et al., 2002; Khan and Das, 2011).

On the other hand, our work suggests an inverse relation between $\mathrm{NO}_{3}$ levels in FF and the potential of embryos to implant in the uterus. Higher $\mathrm{FF} \mathrm{NO}_{3}$ levels were found to be consistent with the presence of nitrotyrosine in granulosa cells, which is indicative of peroxynitrite synthesis (Goud et al., 2014). The synthesis of peroxynitrite takes place when NO interacts with the superoxide anion (Burton and Jauniaux, 2011). The latter species is physiologically produced during folliculogenesis, but lifestyle factors can lead to an unbalance in its regulation (Agarwal et al., 2012). In turn, the peroxynitrite causes lipid peroxidation and cellular damage (Radi et al., 1991). Goud et al. (2014) reported increased $\mathrm{FF} \mathrm{NO}_{3}$ levels in women with versus without endometriosis. Additionally, the affected women who achieved a

TABLE 4 | Association between nitric-oxide-related parameters and the adjusted oocyte yield, number, and proportion of MIl oocytes in of healthy women undergoing ovarian stimulation as part of their participation in an oocyte donation program.

\begin{tabular}{|c|c|c|c|c|}
\hline & n cycles/n donors & Oocyte yield, $\mathrm{n}^{1}$ & MII oocytes, $\mathrm{n}^{1}$ & MII oocyte proportion $(\%)^{2}$ \\
\hline $\mathrm{NO}_{2}$ & $93 / 72$ & & & \\
\hline 1st tertile & $31 / 25$ & $18.2(15.0,22.1)$ & $12.4(10.2,15.1)$ & $68(58,77)$ \\
\hline 2nd tertile & $31 / 23$ & $18.5(15.1,22.6)$ & $14.0(11.4,17.1)$ & $78(69,85)$ \\
\hline 3rd tertile & $31 / 24$ & $18.3(15.1,22.1)$ & $13.2(10.9,16.0)$ & $79(70,85)$ \\
\hline $\mathrm{p}$, linear trend ${ }^{3}$ & & 0.94 & 0.38 & 0.02 \\
\hline $\mathrm{NO}_{3}$ & $93 / 72$ & & & \\
\hline 1st tertile & $30 / 24$ & $18.5(15.4,22.4)$ & $14.1(11.7,17.1)$ & $79(70,85)$ \\
\hline 2nd tertile & $32 / 24$ & $18.1(15.0,21.9)$ & $13.2(10.9,16.1)$ & $78(70,85)$ \\
\hline 3rd tertile & $31 / 24$ & $18.3(15.0,22.5)$ & $12.2(9.9,15.0)$ & $68(57,77)$ \\
\hline $\mathrm{p}$, linear trend ${ }^{3}$ & & 0.88 & 0.14 & 0.03 \\
\hline NOx & $93 / 72$ & & & \\
\hline 1st tertile & $30 / 26$ & $19.2(16.0,23.1)$ & $13.7(11.4,16.5)$ & $74(65,81)$ \\
\hline 2nd tertile & $31 / 20$ & $21.2(17.3,25.8)$ & $15.2(12.4,18.6)$ & $77(68,84)$ \\
\hline 3rd tertile & $32 / 26$ & $16.4(13.7,19.7)$ & $12.2(10.1,14.6)$ & $77(69,84)$ \\
\hline $\mathrm{p}$, linear trend ${ }^{3}$ & & 0.14 & 0.31 & 0.41 \\
\hline $\mathrm{NO}_{3} / \mathrm{NO}_{2}$ ratio & $93 / 72$ & & & \\
\hline 1st tertile & $31 / 23$ & $21.7(16.7,28.1)$ & $14.1(10.7,18.6)$ & $68(52,80)$ \\
\hline 2nd tertile & $31 / 23$ & $16.8(13.8,20.6)$ & $12.9(10.4,15.9)$ & $82(73,88)$ \\
\hline 3rd tertile & $31 / 26$ & $17.2(13.4,22.2)$ & $12.6(9.6,16.5)$ & $74(61,84)$ \\
\hline $\mathrm{p}$, linear trend ${ }^{3}$ & & 0.18 & 0.54 & 0.42 \\
\hline
\end{tabular}

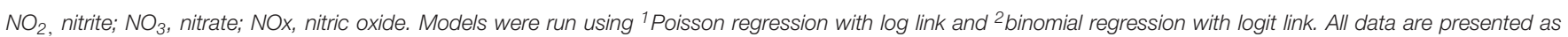

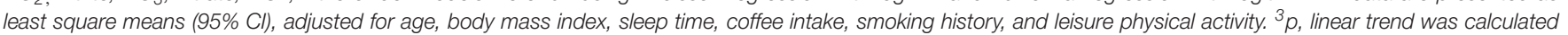
by modeling the tertiles of each metabolite, using the median analyte concentration values in each tertile as a continuous linear term.

TABLE 5 | Summary statistics for embryo development and quality from oocytes of healthy women undergoing ovarian stimulation as part of their participation in an oocyte donation program $(n=75)$.

\begin{tabular}{|c|c|c|c|c|c|c|c|c|}
\hline & \multirow[t]{2}{*}{ Mean (SD) } & \multirow[t]{2}{*}{ Minimum } & \multicolumn{5}{|c|}{ Selected percentiles } & \multirow[t]{2}{*}{ Maximum } \\
\hline & & & 5th & 25th & 50th & 75th & 95th & \\
\hline Number of inseminated oocytes & $13.8(5.1)$ & 6 & 7.8 & 10 & 13 & 16 & 24 & 37 \\
\hline Fertilization rate (\%) & $72.7(16.9)$ & 16.7 & 36.7 & 62.5 & 73.3 & 85.7 & 100 & 100 \\
\hline Number of embryos obtained (cleaved) & $10.1(4.5)$ & 1 & 4.8 & 7 & 9 & 12 & 21.4 & 24 \\
\hline Embryo rate (\%) (cleaved) & $61.8(29.6)$ & 0 & 8.9 & 39.1 & 66.7 & 87.5 & 100 & 100 \\
\hline Number of embryos on day 5 & $6.4(4.6)$ & 0 & 0,8 & 3 & 6 & 8 & 17 & 24 \\
\hline Embryos A + B & $3.8(2.8)$ & 0 & 0 & 2 & 3 & 5 & 9 & 14 \\
\hline Embryos C & $0.9(1.2)$ & 0 & $<1$ & $<1$ & $<1$ & 1 & 3.2 & 5 \\
\hline Embryos D & $1.2(1.6)$ & 0 & $<1$ & $<1$ & 1 & 2 & 5 & 8 \\
\hline
\end{tabular}

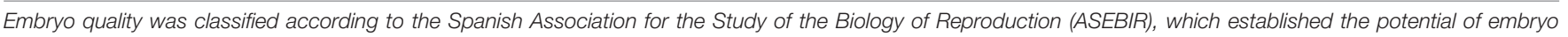
implantation as: A, maximum; B, high; C, medium; D, low (see Table 1). 


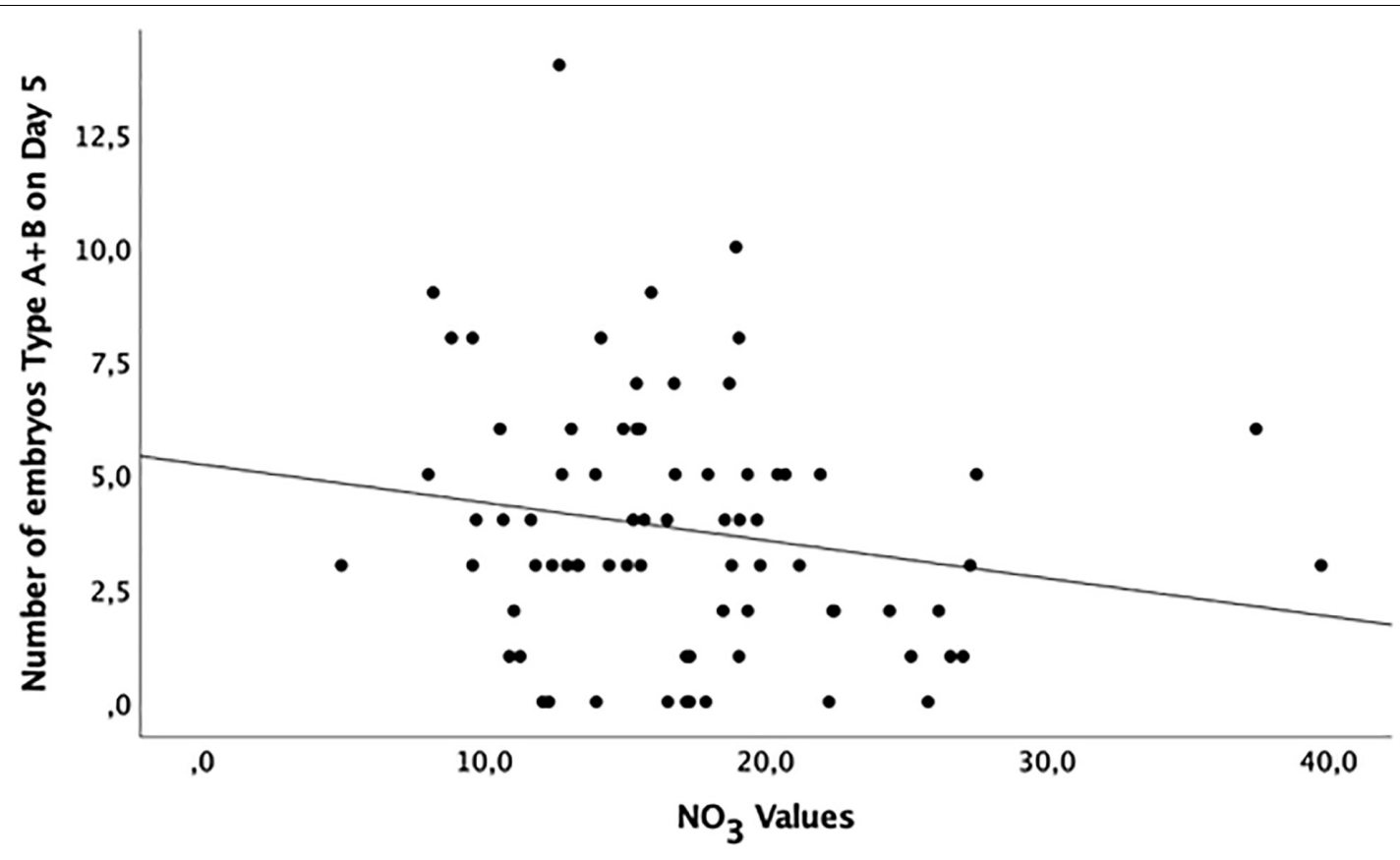

FIGURE 3 | Distribution of grade $\mathrm{A}$ and $\mathrm{B}$ embryos (maximum and high potential of implantation, respectively) at day 5 of development according to the $\mathrm{NO}_{3}$ levels in the follicular fluid of healthy women undergoing ovarian stimulation as part of their participation in an oocyte donation program $(n=75)$.

TABLE 6 | Correlation between $\mathrm{NO}_{2}, \mathrm{NO}_{3}, \mathrm{NOx}$, ratio $\mathrm{NO}_{3} / \mathrm{NO}_{2}$, and embryo development and quality of healthy women undergoing ovarian stimulation as part of their participation in an oocyte donation program $(n=75)$.

\begin{tabular}{|c|c|c|c|c|c|c|c|c|}
\hline & \multicolumn{2}{|c|}{$\mathrm{NO}_{2}$} & \multicolumn{2}{|c|}{$\mathrm{NO}_{3}$} & \multicolumn{2}{|c|}{ NOx } & \multicolumn{2}{|c|}{ Ratio $\mathrm{NO}_{3} / \mathrm{NO}_{2}$} \\
\hline & $r$ & $p$ value & $r$ & $p$ value & $r$ & $p$ value & $r$ & $p$ value \\
\hline Number of inseminated oocytes & 0.05 & 0.68 & -0.12 & 0.29 & -0.01 & 0.92 & -0.07 & 0.57 \\
\hline Fertilization rate (\%) & 0.13 & 0.25 & -0.02 & 0.84 & 0.11 & 0.34 & -0.17 & 0.16 \\
\hline Number of embryos obtained (cleaved) & 0.06 & 0.61 & -0.11 & 0.35 & 0.02 & 0.88 & -0.11 & 0.33 \\
\hline Embryo rate (\%) (cleaved) & -0.05 & 0.65 & -0.20 & 0.08 & -0.18 & 0.11 & -0.02 & 0.87 \\
\hline Number of embryos on day 5 & 0.01 & 0.91 & -0.19 & 0.10 & -0.11 & 0.33 & -0.08 & 0.49 \\
\hline Embryos $A+B$ & 0.08 & 0.50 & -0.20 & 0.07 & -0.08 & 0.51 & -0.15 & 0.29 \\
\hline Embryos C & -0.05 & 0.65 & -0.11 & 0.37 & -0.08 & 0.48 & 0.02 & 0.86 \\
\hline Embryos D & -0.02 & 0.88 & 0.03 & 0.81 & 0.02 & 0.90 & 0.06 & 0.62 \\
\hline
\end{tabular}

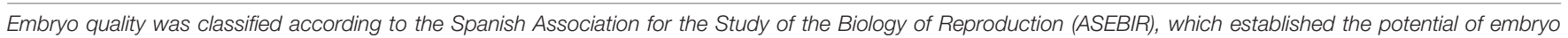
implantation as: A, maximum; B, high; C, medium; D, low (see Table 1). r, Spearman's rank correlation coefficient.

pregnancy had significantly lower $\mathrm{FF} \mathrm{NO}_{3}$ levels. The authors, therefore, suggested that the presence of high concentrations of $\mathrm{NO}_{3}$ and peroxynitrite in the oocyte microenvironment may contribute to a poor follicular health, oocyte quality, embryo quality, and potential embryo implantation. This might justify the negative correlation between the proportion of MII oocytes and embryo quality and the $\mathrm{NO}_{3}$ levels reported in our study.

In conclusion, the direct measurement of NO in biological fluids is problematic because of its short-lived nature. Nonetheless, different techniques can be used to determine $\mathrm{NO}_{2}$ and $\mathrm{NO}_{3}$ levels, but in some cases, it is not possible to simultaneously detect these ions, and complex derivatization procedures might be involved. HPLC-UV represents a valid alternative, as it is a rapid, sensitive, and selective method to detect $\mathrm{NO}_{2}$ and $\mathrm{NO}_{3}$, besides from having already been used in plant and animal samples. In this study, we successfully tested this method with human FF samples, which allowed us to investigate how the $\mathrm{NO}_{2}$ and $\mathrm{NO}_{3}$ levels in this fluid correlate with the ovarian response and embryo quality in human oocyte donors. We detected an association between the FF levels of these species and the proportion of MII oocytes and, possibly, with the quality and implantation potential of embryos derived from those oocytes. However, we have not detected an association between $\mathrm{NO}_{2}$ and $\mathrm{NO}_{3}$ levels in FF and the total and MII oocyte yield. This absence of a correlation with total oocyte counts may reflect a lack of association of the $\mathrm{NO}_{2} / \mathrm{NO}_{3}$-mediated pathway with the ovarian reserve. Although there is no correlation between $\mathrm{NO}_{2}$ and $\mathrm{NO}_{3}$ with the ovarian reserve, their levels could indicate the 
maturation of the oocytes $\left(\mathrm{NO}_{2}\right.$ above all) and embryo quality ( $\mathrm{NO}_{3}$ above all), and both $\mathrm{NO}_{2}$ and $\mathrm{NO}_{3}$ are representative for the formation of NO. It is unclear to what extent differences in MII oocyte proportion and embryo quality due to different FF levels of $\mathrm{NO}_{2}$ or $\mathrm{NO}_{3}$ could impact downstream outcomes like pregnancy and birth rates. Further studies should address these questions in the patients who received the oocytes obtained from our cohort of donors.

\section{DATA AVAILABILITY STATEMENT}

The raw data supporting the conclusions of this article will be made available by the authors, without undue reservation.

\section{ETHICS STATEMENT}

The studies involving human participants were reviewed and approved by the Ethics Review Committee of CEIC Hospital General Universitario Jose Maria Morales Meseguer (Murcia, Spain) (Approval No. EST: 06/17) and registered at https:// clinicaltrials.gov/ (ID: NCT03307655). Women participating in the gamete donation program at IVI-RMA Global (Murcia, Spain) were invited to participate in the study. All the donors who accepted provided their written informed consent. The patients/participants provided their written informed consent to participate in this study.

\section{AUTHOR CONTRIBUTIONS}

F-DS recruited the oocyte donors, analyzed the data, and wrote the manuscript. AC-G recruited the oocyte donors, analyzed the data, and reviewed the manuscript. CS-Ú and EA analyzed the

\section{REFERENCES}

Agarwal, A., and Allamaneni, S. S. R. (2004). Role of free radicals in female reproductive diseases and assisted reproduction. Reprod. Biomed. Online 9, 338-347. doi: 10.1016/s1472-6483(10)62151-7

Agarwal, A., Aponte-Mellado, A., Premkumar, B. J., Shaman, A., and Gupta, S. (2012). The effects of oxidative stress on female reproduction: a review. Reprod. Biol. Endocrinol. 10:49. doi: 10.1186/1477-7827-10-49

Anteby, E. Y., Hurwitz, A., Korach, O., Revel, A., Simon, A., Finci-Yeheskel, Z., et al. (1996). Human follicular nitric oxide pathway: relationship to follicular size, oestradiol concentrations and ovarian blood flow. Hum. Reprod. 11, 1947-1951. doi: 10.1093/oxfordjournals.humrep.a019522

Barrionuevo, M. J., Schwandt, R. A., Rao, P. S., Graham, L. B., Maisel, L. P., and Yeko, T. R. (2000). Nitric oxide (NO) and interleukin-1beta (IL-1beta) in follicular fluid and their correlation with fertilization and embryo cleavage. Am. J. Reprod. Immunol. 44, 359-364. doi: 10.1111/j.8755-8920.2000.440607.x

Barroso, G., Barrionuevo, M., Rao, P., Graham, L., Danforth, D., Huey, S., et al. (1999). Vascular endothelial growth factor, nitric oxide, and leptin follicular fluid levels correlate negatively with embryo quality in IVF patients. Fertil. Steril. 72, 1024-1026. doi: 10.1016/s0015-0282(99)00442-2

Basini, G., Baratta, M., Ponderato, N., Bussolati, S., and Tamanini, C. (1998). Is nitric oxide an autocrine modulator of bovine granulosa cell function? Reprod. Fertil. Dev. 10, 471-478. doi: 10.1071/rd98114

Battaglia, C., Persico, N., Mancini, F., De Iaco, P., Busacchi, P., Facchinetti, F., et al. (2006). Uterine vascularization and pregnancy outcome in patients undergoing data and wrote the manuscript. JM-S recruited the oocyte donors, provided the clinical and lifestyle-related data, and reviewed the manuscript. JC designed the statistical analysis, analyzed the data, and reviewed the manuscript. CM conceived and designed the study and reviewed the manuscript. All authors read and approved the final manuscript.

\section{FUNDING}

This work has received funding from the European Union's H2020 research and innovation program under the Marie Skłodowska-Curie Actions grant agreement Rep-Biotech 675526 and from the Spanish Ministry of Science and Innovation PID2019-106380RB-I00/AEI/10.13039/501100011033.

\section{ACKNOWLEDGMENTS}

The authors would like to thank all the staff from the Embryology Laboratory at IVI-RMA Global (Murcia, Spain) for their assistance in recruiting the FF donors. Furthermore, the authors thank Prof. Joaquín Ortuño Sánchez-Pedreño and Dr. Alejandro Torrecillas for their help with the $\mathrm{NO}_{2}$ and $\mathrm{NO}_{3}$ measurements, Prof. Javier Corral de la Calle for providing the HemoCue Plasma/Low Hb System, and Prof. Dr. Jaime Mendiola Olivares for his assistance with the statistical analyses.

\section{SUPPLEMENTARY MATERIAL}

The Supplementary Material for this article can be found online at: https://www.frontiersin.org/articles/10.3389/fcell.2021. 647002/full\#supplementary-material

intracytoplasmatic sperm injection: the role of nitric oxide. J. Assist. Reprod. Genet. 23, 213-222. doi: 10.1007/s10815-006-9049-x

Burton, G. J., and Jauniaux, E. (2011). Oxidative stress. Best Pract. Res. Clin. Obstet. Gynaecol. 25, 287-299. doi: 10.1016/j.bpobgyn.2010. 10.016

Cigliano, L., Balestrieri, M., Spagnuolo, M. S., Dale, B., and Abrescia, P. (2002). Lecithin-cholesterol acyltransferase activity during maturation of human preovulatory follicles with different concentrations of ascorbate, alphatocopherol and nitrotyrosine. Reprod. Fertil. Dev. 14, 15-21. doi: 10.1071/ rd01044

Croitoru, M. D. (2012). Nitrite and nitrate can be accurately measured in samples of vegetal and animal origin using an HPLC-UV/VIS technique. J. Chromatogr. B. Analyt. Technol. Biomed. Life Sci. 911, 154-161. doi: 10.1016/j.jchromb.2012. 11.006

Cuevas Saiz, I., Carme Pons Gatell, M., Vargas, M. C., Delgado Mendive, A., Rives Enedáguila, N., Moragas Solanes, M., et al. (2018). The Embryology Interest Group: updating ASEBIR's morphological scoring system for early embryos, morulae and blastocysts. Med. Reprod. Embriol. Clín. 5, 42-54. doi: 10.1016/ j.medre.2017.11.002

Gentry, P. A., Plante, L., Schroeder, M. O., LaMarre, J., Young, J. E., and Dodds, W. G. (2000). Human ovarian follicular fluid has functional systems for the generation and modulation of thrombin. Fertil. Steril. 73, 848-854. doi: 10. 1016/s0015-0282(99)00635-4

Gonzalès, J., Lesourd, S., Van Dreden, P., Richard, P., Lefèbvre, G., and Vauthier Brouzes, D. (1992). Protein composition of follicular fluid and oocyte cleavage 
occurrence in in vitro fertilization (IVF). J. Assist. Reprod. Genet. 9, 211-216. doi: $10.1007 /$ bf01203815

Goud, A. P., Goud, P. T., Diamond, M. P., and Abu-Soud, H. M. (2005). Nitric oxide delays oocyte aging. Biochemistry 44, 11361-11368. doi: 10.1021/ bi050711f

Goud, P. T., Goud, A. P., Joshi, N., Puscheck, E., Diamond, M. P., and Abu-Soud, H. M. (2014). Dynamics of nitric oxide, altered follicular microenvironment, and oocyte quality in women with endometriosis. Fertil. Steril. 102, 151.e5159.e5. doi: 10.1016/j.fertnstert.2014.03.053

Gulamali-Majid, F., Ackerman, S., Veeck, L., Acosta, A., and Pleban, P. (1987). Kinetic immunonephelometric determination of protein concentrations in follicular fluid. Clin. Chem. 33, 1185-1189. doi: 10.1093/clinchem/33.7.1185

Hakim, T. S., Sugimori, K., Camporesi, E. M., and Anderson, G. (1996). Half-life of nitric oxide in aqueous solutions with and without haemoglobin. Physiol. Meas. 17, 267-277. doi: 10.1088/0967-3334/17/4/004

Hurtado de Mendoza, M., Cuadros, J., Arroyo, G., Ten, J., Pons, M., Prados, F., et al. (2015). Cuadernos de Embriología clínica. Criterios ASEBIR de Valoración Morfológica De Oocitos, Embriones Tempranos y Blastocistos Humanos, 3rd Edn. Gipuzkoa: ASEBIR.

Khan, F. A., and Das, G. K. (2011). Follicular fluid nitric oxide and ascorbic acid concentrations in relation to follicle size, functional status and stage of estrous cycle in buffalo. Anim. Reprod. Sci. 125, 62-68. doi: 10.1016/j.anireprosci.2011. 03.012

Kim, K. H., Oh, D. S., Jeong, J. H., Shin, B. S., Joo, B. S., and Lee, K. S. (2004). Follicular blood flow is a better predictor of the outcome of in vitro fertilizationembryo transfer than follicular fluid vascular endothelial growth factor and nitric oxide concentrations. Fertil. Steril. 82, 586-592. doi: 10.1016/j.fertnstert 2004.02.120

Lee, K. S., Joo, B. S., Na, Y. J., Yoon, M. S., Choi, O. H., and Kim, W. W. (2000). Relationships between concentrations of tumor necrosis factor-alpha and nitric oxide in follicular fluid and oocyte quality. J. Assist. Reprod. Genet. 17, 222-228.

Lidder, S., and Webb, A. J. (2013). Vascular effects of dietary nitrate (as found in green leafy vegetables and beetroot) via the nitrate-nitrite-nitric oxide pathway. Br. J. Clin. Pharmacol. 75, 677-696. doi: 10.1111/j.1365-2125.2012.04420.x

Lundberg, J. O., Weitzberg, E., and Gladwin, M. T. (2008). The nitrate-nitritenitric oxide pathway in physiology and therapeutics. Nat. Rev. Drug Discov. 7, 156-167. doi: 10.1038/nrd2466

Manau, D., Balasch, J., Jiménez, W., Fábregues, F., Civico, S., Casamitjana, R., et al. (2000). Follicular fluid concentrations of adrenomedullin, vascular endothelial growth factor and nitric oxide in IVF cycles: relationship to ovarian response. Hum. Reprod. 15, 1295-1299. doi: 10.1093/humrep/15.6.1295

Melo, M., Busso, C. E., Bellver, J., Alama, P., Garrido, N., Meseguer, M., et al. (2009). $\mathrm{GnRH}$ agonist versus recombinant HCG in an oocyte donation programme: a randomized, prospective, controlled, assessor-blind study. Reprod. Biomed. Online 19, 486-492. doi: 10.1016/j.rbmo.2009.06.001

Pacher, P., Beckman, J. S., and Liaudet, L. (2007). Nitric oxide and peroxynitrite in health and disease. Physiol. Rev. 87, 315-424. doi: 10.1152/physrev.00029. 2006

Panagiotakos, D. B., Pitsavos, C., Arvaniti, F., and Stefanadis, C. (2007). Adherence to the Mediterranean food pattern predicts the prevalence of hypertension, hypercholesterolemia, diabetes and obesity, among healthy adults; the accuracy of the MedDietScore. Prev. Med. 44, 335-340. doi: 10.1016/j.ypmed.2006. 12.009

Radi, R., Beckman, J. S., Bush, K. M., and Freeman, B. A. (1991). Peroxynitriteinduced membrane lipid peroxidation: the cytotoxic potential of superoxide and nitric oxide. Arch. Biochem. Biophys. 288, 481-487. doi: 10.1016/00039861(91)90224-7

Ratajczak-Wrona, W., Jablonska, E., Antonowicz, B., Dziemianczyk, D., and Grabowska, S. Z. (2013). Levels of biological markers of nitric oxide in serum of patients with squamous cell carcinoma of the oral cavity. Int. J. Oral Sci. 5, 141-145. doi: 10.1038/ijos.2013.59

Revelli, A., Delle Piane, L., Casano, S., Molinari, E., Massobrio, M., and Rinaudo, P. (2009). Follicular fluid content and oocyte quality: from single biochemical markers to metabolomics. Reprod. Biol. Endocrinol. 7:40. doi: 10.1186/14777827-7-40

Romero-Aguirregomezcorta, J., Santa, ÁP., García-Vázquez, F. A., Coy, P., and Matás, C. (2014). Nitric oxide synthase (NOS) inhibition during porcine in vitro maturation modifies oocyte protein S-nitrosylation and in vitro fertilization. PLoS One 9:e115044. doi: 10.1371/journal.pone.0115044

Romitelli, F., Santini, S. A., Chierici, E., Pitocco, D., Tavazzi, B., Amorini, A. M., et al. (2007). Comparison of nitrite/nitrate concentration in human plasma and serum samples measured by the enzymatic batch Griess assay, ion-pairing HPLC and ion-trap GC-MS: the importance of a correct removal of proteins in the Griess assay. J. Chromatogr. B Anal. Technol. Biomed. Life Sci. 851, 257-267. doi: 10.1016/j.jchromb.2007.02.003

Rosselli, M., Keller, P. J., and Dubey, R. K. (1998). Role of nitric oxide in the biology, physiology and pathophysiology of reproduction. Hum. Reprod. Update 4, 3-24. doi: 10.1093/humupd/4.1.3

Shiva, S., Wang, X., Ringwood, L. A., Xu, X., Yuditskaya, S., Annavajjhala, V., et al. (2006). Ceruloplasmin is a NO oxidase and nitrite synthase that determines endocrine NO homeostasis. Nat. Chem. Biol. 2, 486-493. doi: 10. 1038/nchembio813

Suchanek, E., Mujkic-Klaric, A., Grizelj, V., Simunic, V., and Kopjar, B. (1990). Protein concentration in pre-ovulatory follicular fluid related to ovarian stimulation. Int. J. Gynaecol. Obstet. 32, 53-59. doi: 10.1016/0020-7292(90) 90982-q

Vignini, A., Turi, A., Giannubilo, S. R., Pescosolido, D., Scognamiglio, P., Zanconi, S., et al. (2008). Follicular fluid nitric oxide (NO) concentrations in stimulated cycles: the relationship to embryo grading. Arch. Gynecol. Obstet. 277, 229-232. doi: 10.1007/s00404-007-0445-y

Yalçınkaya, E., Cakıroğlu, Y., Doğer, E., Budak, O., Cekmen, M., and Calıșkan, E. (2013). Effect of follicular fluid NO, MDA and GSH levels on in vitro fertilization outcomes. J. Turk. Ger. Gynecol. Assoc. 14, 136-141. doi: 10.5152/ jtgga.2013.53323

Zhao, M., Chang, C., Liu, Z., Chen, L. M., and Chen, Q. (2010). The level of vascular endothelial cell growth factor, nitric oxide, and endothelin-1 was correlated with ovarian volume or antral follicle counts: a potential predictor of pregnancy outcome in IVF. Growth Factors 28, 299-305. doi: 10.3109/08977191003766866

Conflict of Interest: The authors declare that the research was conducted in the absence of any commercial or financial relationships that could be construed as a potential conflict of interest.

Copyright (C) 2021 Staicu, Canha-Gouveia, Soriano-Úbeda, Martínez-Soto, Adoamnei, Chavarro and Matás. This is an open-access article distributed under the terms of the Creative Commons Attribution License (CC BY). The use, distribution or reproduction in other forums is permitted, provided the original author(s) and the copyright owner(s) are credited and that the original publication in this journal is cited, in accordance with accepted academic practice. No use, distribution or reproduction is permitted which does not comply with these terms. 